\title{
Microarray based analysis of gene regulation by microRNA in intervertebral disc degeneration
}

\author{
PENG HU $^{1,2}$, BO FENG $^{3}$, GUANGLIN WANG $^{2}$, BIN NING $^{1}$ and TANGHONG JIA ${ }^{1}$ \\ ${ }^{1}$ Department of Orthopedics, Jinan Central Hospital of Shandong University, Jinan, Shandong 250012; \\ Departments of ${ }^{2}$ Spine and ${ }^{3}$ Neurology, Hospital Affiliated to Binzhou Medical University, \\ Binzhou, Shandong 256603, P.R. China
}

Received September 29, 2014; Accepted June 11, 2015

DOI: $10.3892 / \mathrm{mmr} .2015 .4022$

\begin{abstract}
The present study aimed to explore the underlying mechanism of the development of intervertebral disc degeneration (IDD) by bioinformatics based on microarray datasets. GSE 19943 and GSE 34095 datasets downloaded from Gene Expression Omnibus data were used to screen the differentially expressed genes (DEGs) in IDD. The correlation between microRNAs and target genes was investigated using different algorithms. The underlying molecular mechanisms of the target genes were then explored using Kyoto Encyclopedia of Genes and Genomes pathway and Gene Ontology function enrichment analysis. A total of 9 differentially expressed microRNAs, including 3 down- and 6 upregulated microRNAs and 850 DEGs were identified in tissue from patients with IDD. Two regulation networks of the target genes by microRNAs were constructed, including 33 upregulated microRNA-target gene pairs and 4 downregulated microRNA-target gene pairs. Certain target genes had been demonstrated to be involved in IDD progression via various pathways, including in the cell cycle and pathways in cancer. In addition, two important microRNAs (microRNA-222 and microRNA-589) were identified that were pivotal for the development of IDD, and their target genes, $C D K N A B$ and SMAD4. In conclusion, a comprehensive miRNA-target gene regulatory network was constructed, which was found to be important in IDD progression.
\end{abstract}

\section{Introduction}

Intervertebral disc degeneration (IDD) is characterized by pathological changes in the intervertebral disc, and is associated with the development of lower back pain and acute

Correspondence to: Dr Tanghong Jia, Department of Orthopedics, Jinan Central Hospital of Shandong University, 105 Jiefang Road, Jinan, Shandong 250012, P.R. China

E-mail: jiatred@163.com

Key words: intervertebral disc degeneration, microRNA, target genes lumbar radiculopathy (1). It is widely accepted that a number of factors, such as genetic predisposition, abnormal biomechanical loading, ageing and decreased nutrient transport could result in the development of IDD $(2,3)$. However, the underlying mechanism associated with IDD remains largely unknown.

MicroRNAs (miRNAs) are small (19-24 nt), non-protein-coding nucleic acids that regulate specific target gene products via binding to the 3 ' untranslated regions (UTRs) of mRNA transcripts, contributing to mRNA degradation or translational blockade (4). To date, $>1,000$ human miRNAs have been experimentally identified, and it is estimated that they regulate more than one-third of cellular mRNA and are involved in a number of diseases processes (5). Emerging evidence has strongly suggested that abnormal miRNA expression is a common and important feature in IDD. For example, a study by Zhao et al (6) identified 25 upregulated miRNAs and 26 downregulated miRNAs in the IDD via microarray analysis and reverse transcription-quantitative polymerase chain reaction (RT-qPCR). Similarly, 29 miRNAs were found to be differentially expressed in degenerative nucleus pulposus (NP), and deregulated miRNA-155 promoted Fas-mediated apoptosis through targeting caspase-3 and fas-associated death domain containing protein (7). In addition, miRNA-34a induced by 553 lead to cell apoptosis resulting in the development of osteoarthritis (8). In addition, a previous study demonstrated that miRNA-140 expression is associated with chondrocyte differentiation, and therefore contributes to the development of osteoarthritis (9).

Since mRNA is an important intermediary agent for miRNA action, the interaction between miRNA and mRNA is important in the development of complex diseases. Therefore, the objective of this study was to comprehensively investigate the IDD-related gene regulation by constructing miRNA-target gene networks. Furthermore, their underlying molecular mechanisms were also characterized by function annotation and Kyoto Encyclopedia of Genes and Genomes (KEGG) pathway enrichment analysis.

\section{Materials and methods}

Affymetrix microarray data. GSE 19943 (7) and GSE 34095 datasets were accessible at the National Center for 
Biotechnology Information Gene Expression Omnibus database (http://www.ncbi.nlm.nih.gov/geo/). The miRNA and mRNA microarray datasets are based on the platforms of GPL9964-Exiqon human miRCURY LNA ${ }^{\mathrm{TM}}$ miRNA Array V11.0 (Exiqon, Vedbaek, Denmark) and GPL96-[HG-U133A] Affymetrix Human Genome U133A Array (Affymetrix, Santa Clara, CA, USA), respectively. miRNA expression profiling of human NP cells derived from 3 patients with disc degeneration compared with 3 controls with scoliosis. Similarly, mRNA datasets included human intervertebral disc tissues harvested from 3 elderly and 3 younger patients with degenerative disc disease and adolescent idiopathic scoliosis, respectively.

Data processing and DEG screen. The correlation between miRNA and the target gene was determined based on the original expression profiling, and $\log _{2}$-transformed microarray data was used to screen the differentially expressed genes (DEGs) in intervertebral disc tissues from patients with degenerative disc disease and adolescent idiopathic scoliosis by Linear Models for Microarray Data (LIMMA) package in $\mathrm{R}$ (10). False discovery rates (FDRs) were calculated using the method of Benjamini and Hochberg (11). FDR-corrected $\mathrm{P}<0.05$ and $\mid \log$ (fold change) $\mid>1$ were defined as the thresholds for screening DEGs.

Differentially expressed analysis of miRNA and the target genes. miRNA and mRNA integrated analysis (MMIA) is a versatile web serverintegrating miRNA and mRNA expression data with predicted miRNA target information for analyzing miRNA-associated phenotypes and biological functions by gene set analysis (12). Target genes of differentially expressed miRNA were screened by combining TargetScan 5.1 (http://www.targetscan.org; Whitehead Institute for Biomedical Research, Cambridge, MA, USA) and Pictar4way algorithms (http://pictar.mdc-berlin.de; Center for Comparative Functional Genomics and the Max Delbruck Centrum, Berlin, Germany). To explore the association between miRNA and DEGs in the development of IDD, the enrolled DEGs were then further screened to analyze the miRNA and the target DEGs in human intervertebral disc tissue.

Regulation association analysis and network construction. The software of Search Tool for the Retrieval of Interacting Genes (STRING; http://string-db.org/) is a tool that could cluster networks on demand, update on-screen previews of structural information including homology models and extensive data updates (13). In order to correctly uncover and annotate all functional interactions among proteins in the human NP cells, STRING was employed to determine the correlation between the differentially expressed miRNA and DEGs in the IDD process. A confidence score was employed to define the interaction between miRNA and DEGs based on experimental measurements and computational prediction techniques. The confidence score $>0.4$ was defined statistically significant. Cytoscape (http://cytoscape.org/) is a popular bioinformatics package for biological network visualization and data integration (14). The interaction network was visualized by the Cytoscape tool based on the above association.
Function annotation and pathway analysis. The Biological Networks Gene Ontology tool (BiNGO; http://www.psb.ugent.be/cbd/papers/BiNGO/), as a cytoscape plugin, is an open-source Java tool to determine which Gene Ontology (GO) terms are significantly overrepresented in a set of genes (15). BiNGO was used to perform functional annotation for the network with a threshold of adjusted $\mathrm{P}<0.05$ based on hypergeometric distribution. Web-based Gene Set Enrichment Analysis Toolkit (WebGestalt; http://www.webgestalt.org) is composed of four modules: Gene set management, information retrieval, organization/visualization and statistics, and shifted the study from 'single genes' to 'gene sets' $(16,17)$. WebGestalt was used to perform KEGG pathway analysis for DEGs regulated by differentially expressed miRNA in IDD tissue and $\mathrm{P}<0.05$ was considered to indicate a statistically significant difference.

\section{Results}

Differentially expressed miRNA and $m R N A$ analysis. A total of 253 miRNAs and their associated 22,2215 genes were obtained based on their correlation between the miRNAs and associated genes of the original expression profile. Differentially expressed analyses of miRNA and mRNA were performed between cases and control by LIMMA package in $\mathrm{R}$ language and multiple comparisons using FDR and Benjamini and Hochberg after normalization (Fig. 1). A total of 850 DEGs and 9 miRNAs, including 3 up- and 6 downregulated miRNAs (Table I), were identified to be significantly expressed with thresholds of $\mid \log _{2} \mathrm{FCl}>1$ and FDR-corrected $\mathrm{P}<0.05$.

miRNA-target gene regulation association. Target genes of nine differentially expressed miRNAs were obtained from the TargetScan database, and the abnormally expressed miRNAs were then screened based on conservative property at the 3'UTR and context score. Among them, target genes of 8 abnormally expressed miRNAs were found to be differentially expressed. As shown in Table I, a total of 8 miRNAs and 37 target DEGs were screened. No predicted target genes were identified for miRNA-30c-1.

miRNA-DEG regulation network construction. To explore the regulation network for the target genes regulated by miRNAs, the correlation between proteins encoded by DEGs was identified by mapping both up- and downregulated DEGs to STRING. A total of 4 interaction pairs were constructed with a confidence score $>0.4$, including interaction relationships of SMAD family member 4 (SMAD4) and activin A receptor, type IB, WW domain containing adaptor with coiled-coil and bromodomain adjacent to zinc finger domain, 1B, BMI1 polycomb ring finger oncogene and cyclin-dependent kinase inhibitor 1B (CDKN1B), SMAD4 and CDKN1B. As shown in (Fig. 2), the interactions were visualized by Cytoscape. The network included 8 miRNA and 37 target DEGs, including miRNA-222 targeting the gene set of ataxin 1, BMI1, CDKN1B and defective in cullin neddylation 1, domain containing 4 and miRNA-589 targeting the gene set of F-box and WD repeat domain containing 2, G-rich RNA sequence binding factor 1, protein kinase N2 and SMAD4. 
Table I. Differentially expressed miRNAs in intervertebral disc degeneration tissue and the target genes.

\begin{tabular}{lllll}
\hline miRNA_ID_LIST & ID & FDR & $\operatorname{logFC}$ & \multicolumn{1}{c}{ Target genes } \\
\hline hsa-miR-30c-1 a & 1158 & 0.0315 & -3.01 & - \\
hsa-miR-638 & 1151 & 0.0483 & -2.254 & PAK2 \\
hsa-miR-1275 & 1223 & 0.0387 & -2.252 & SERBP1, BAIAP2, USF2 \\
hsa-miR-589 & 1104 & 0.0407 & 2.5 & FBXW2, GRSF1, PKN2, SMAD4 \\
hsa-miR-1286 & 1026 & 0.0387 & 2.767 & ABCF2, BACH2, BAZ1B, CELSR2, \\
& & & SYP, VAMP1 \\
hsa-miR-222 & 1012 & 0.0315 & 3.05 & ATXN1, BMI1, CDKN1B, DCUN1D4, \\
& & & GTF2E1, KIAA0368, NAP1L1, NFYB, \\
hsa-miR-220b & 1122 & 0.0387 & PAIP1, T1MP3, TRAM2 \\
hsa-miR-640 & 1030 & 0.0132 & 4.216 & ACVR1B, ARL4C, FAM20B, SMYD5 \\
hsa-miR-532-3p & 1060 & 0.0315 & 4.559 & GRIK2, TNP01
\end{tabular}

miRNA, microRNA; FDR, false discovery rate.

$\mathbf{A}$

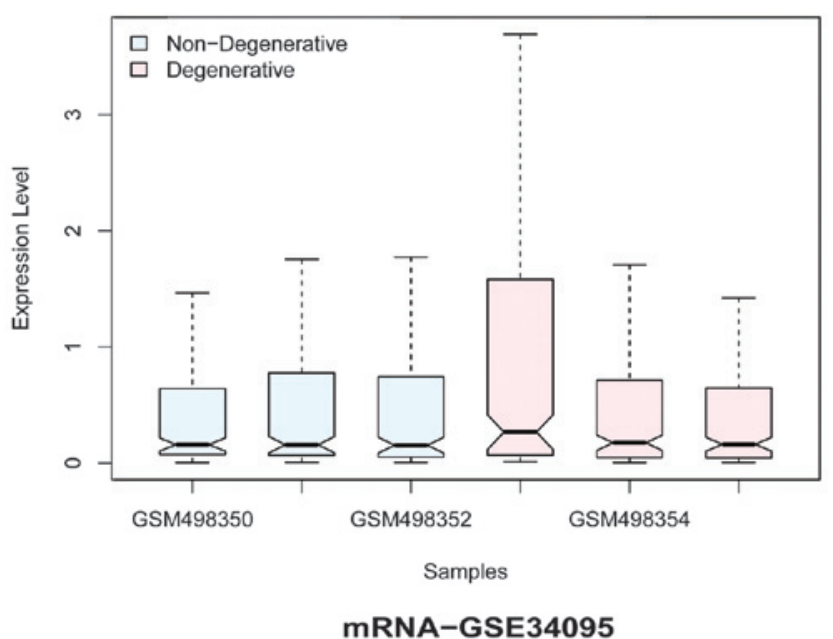

B

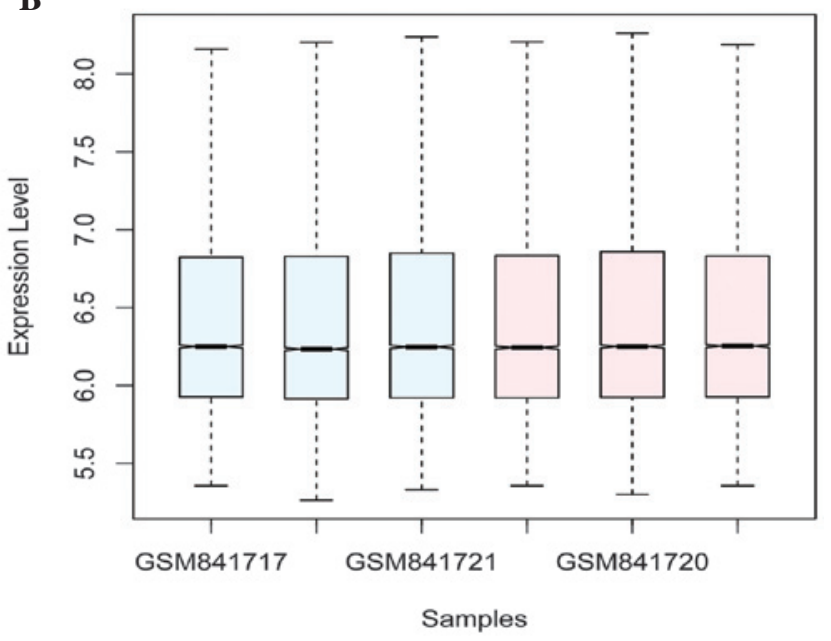

Figure 1. Box plots for miRNA and mRNA microarray datasets after normalization. (A) Box plot for miRNA microarray dataset after normalization; (B) box plot for mRNA microarray dataset after normalization. miRNA, microRNA.
Function and pathway annotation. miRNAs regulate specific target gene products via hybridization to mRNA, the 8 miRNAs regulated 37 common target genes, which may be important in the development of IDD. Therefore, the present study used function annotation and pathway analysis for the DEGs regulated by the differential miRNAs in human NP cells. As shown in Table II, the top $10 \mathrm{GO}$ biological processes were identified for DEGs involved in upregulated miRNA networks, including negative regulation of cell growth, negative regulation of cell size and negative regulation of growth, and DEGs associated with downregulated miRNAs, such as positive regulation of transcription by glucose, positive regulation of transcription from the RNA polymerase II promoter by glucose and positive of transcription from RNA polymerase II promoter by carbon catabolites, based on the hypergeometric test and $\mathrm{P}<0.05$ (Table II). Two KEGG pathways were screened using the WebGestalt tool according to adjusted $\mathrm{P}<0.05$, the cell cycle and pathways in cancer (Table III). Notably, the two pathways involved CDKN1B and SMAD4.

\section{Discussion}

In an effort to gain greater understanding of the mechanism underlying IDD, miRNA and mRNA datasets were downloaded to explore the IDD-related genes and an miRNA regulation network was constructed to investigate the potential mechanism in the development of IDD. Functional annotation showed that the upregulated miRNA predominantly regulated pathways associated with cell activity, such as the negative regulation of cell growth, cell size and cell death, and downregulated miRNAs predominantly regulated pathways associated with the regulation of transcription by glucose and the regulation of transcription by carbon catabolites. In the present study, the differentially expressed miRNAs were predicted to control 2 pathways relevant to IDD, including the cell cycle and pathways in cancer. Notably, the pathways were regulated 
Table II. Gene functional annotation for up- and downregulated miRNA-target genes networks.

A, Upregulated miRNA network

\begin{tabular}{|c|c|c|c|}
\hline GO-ID & Adj P-value & Description & Gene sets \\
\hline 30308 & 0.044851 & Negative regulation of cell growth & ACVR1B, CDKN1B, SMAD4 \\
\hline 45792 & 0.044851 & Negative regulation of cell size & ACVR1B, CDKN1B, SMAD4 \\
\hline 45926 & 0.044851 & Negative regulation of growth & ACVR1B, CDKN1B, SMAD4 \\
\hline 12502 & 0.046347 & Induction of programmed cell death & ACVR1B, CDKN1B, GRIK2, TIMP3 \\
\hline 60255 & 0.046347 & $\begin{array}{l}\text { Regulation of macromolecule } \\
\text { metabolic process }\end{array}$ & $\begin{array}{l}\text { BMI1, BACH2, PAIP1, SMAD4, } \\
\text { NFYB, CELSR2, FOXJ3, ATXN1, } \\
\text { GTF2E1, ACVR1B, LBH, } \\
\text { CDKN1B, BAZ1B, PBX2 }\end{array}$ \\
\hline 31323 & 0.046347 & Regulation of cellular metabolic process & $\begin{array}{l}\text { BMI1, BACH2, PAIP1, SMAD4, } \\
\text { NFYB, CELSR2, FOXJ3, ATXN1, } \\
\text { GTF2E1, ACVR1B, LBH, } \\
\text { CDKN1B, BAZ1B, PBX2 }\end{array}$ \\
\hline 19219 & 0.046347 & $\begin{array}{l}\text { Regulation of nucleobase, nucleoside, } \\
\text { nucleotide and nucleic acid } \\
\text { metabolic process }\end{array}$ & $\begin{array}{l}\text { BMI1, BACH2, PAIP1, SMAD4, NFYB, } \\
\text { CELSR2, FOXJ3, ATXN1, GTF2E1, } \\
\text { ACVR1B, LBH, BAZ1B, PBX2 }\end{array}$ \\
\hline 19222 & 0.046347 & Regulation of metabolic process & $\begin{array}{l}\text { BMI1, BACH2, PAIP1, SMAD4, } \\
\text { NFYB, CELSR2, FOXJ3, ATXN1, } \\
\text { GTF2E1, ACVR1B, LBH, } \\
\text { CDKN1B, BAZ1B, PBX2 }\end{array}$ \\
\hline 10556 & 0.046347 & Regulation of macromolecule & $\begin{array}{l}\text { BMI1, BACH2, PAIP1, SMAD4, NFYB, } \\
\text { CELSR2, FOXJ3, ATXN1, GTF2E1, } \\
\text { ACVR1B, LBH, BAZ1B, PBX2 }\end{array}$ \\
\hline 8361 & 0.046347 & Regulation of cell size & ACVR1B, CDKN1B, SMAD4 \\
\hline
\end{tabular}

B, Downregulated miRNA network

\begin{tabular}{|c|c|c|c|}
\hline GO-ID & Adj P-value & Description & Gene set \\
\hline 46016 & 0.011654 & Positive regulation of transcription by glucose & USF2 \\
\hline 432 & 0.011654 & $\begin{array}{l}\text { Positive regulation of transcription from RNA polymerase II } \\
\text { promoter by glucose }\end{array}$ & USF2 \\
\hline 436 & 0.011654 & $\begin{array}{l}\text { Positive regulation of transcription from RNA polymerase II } \\
\text { promoter by carbon catabolites }\end{array}$ & USF2 \\
\hline 429 & 0.011654 & $\begin{array}{l}\text { Regulation of transcription from RNA polymerase II } \\
\text { promoter by carbon catabolites }\end{array}$ & USF2 \\
\hline 430 & 0.011654 & $\begin{array}{l}\text { Regulation of transcription from RNA polymerase II } \\
\text { promoter by glucose }\end{array}$ & USF2 \\
\hline 45991 & 0.011654 & Positive regulation of transcription by carbon catabolites & USF2 \\
\hline 46015 & 0.01311 & Regulation of transcription by glucose & USF2 \\
\hline 45990 & 0.01311 & Regulation of transcription by carbon catabolites & USF2 \\
\hline 43488 & 0.017476 & Regulation of mRNA stability & SERBP1 \\
\hline 31670 & 0.017476 & Cellular response to nutrient & USF2 \\
\hline
\end{tabular}

miRNA, microRNA; GO, gene ontology.

by CDKN1B and SMAD4 genes. Furthermore, CDKN1B and SMAD4 were shown to exhibit a significant interaction and were regulated by miRNA-222 and miRNA-589, respectively.

The disc is composed of the central highly hydrated NP, the surrounding elastic and fibrous annulus fibrosus (AF), and the cartilaginous endplate (CEP), and each of these tissues has a different function and consists of a specific matrix structure that is maintained by a cell population with a distinct phenotype. Disturbed homeostasis of these tissues may lead to pathological conditions, including pathologies associated with cellular phenotype and biochemical factors (18). In accordance with the study by Wang et al (7), Fas-mediated 
A

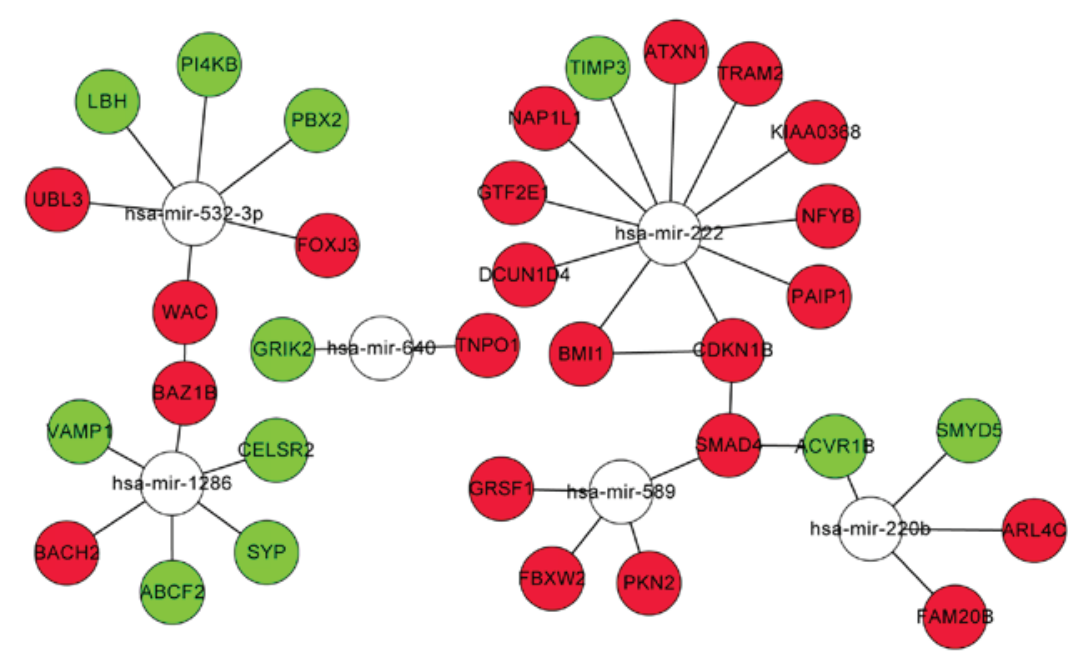

B

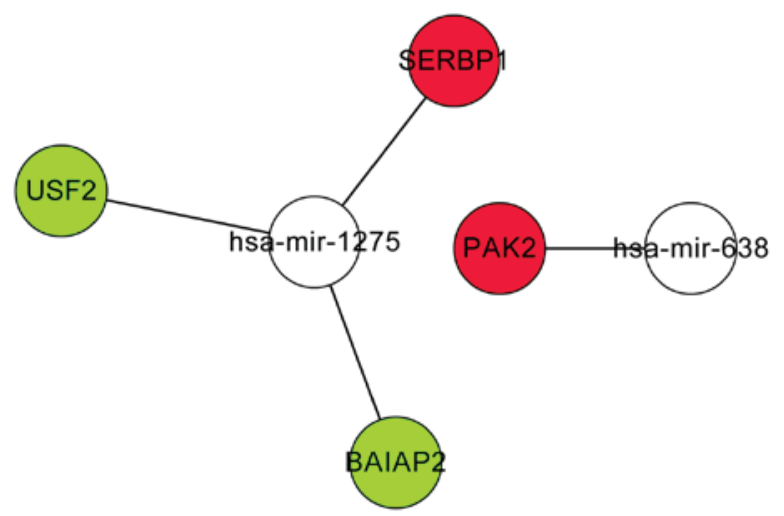

Figure 2. Up- and downregulated miRNA-target genes network. (A) Upregulated miRNA-target genes network; (B) downregulated miRNA-target genes network. miRNA, microRNA.

Table III. Kyoto encyclopedia of genes and genomes pathways for differentially expressed genes regulated by miRNAs.

\begin{tabular}{lcc}
\hline Pathway & Adj P-value & Gene sets \\
\hline Cell cycle & 0.0062 & CDKN1B, SMAD4 \\
Pathways in cancer & 0.0258 & CDKN1B, SMAD4 \\
\hline
\end{tabular}

apoptosis in human IDD triggered by miRNA-155 via targeting Fas-associated death domain containing protein and caspase- 3 contributes to IDD. In the current study, the upregulated miRNA predominantly regulated pathways associated with cell activity, such as the negative regulation of cell growth, cell size and cell death. Moreover, the critical roles of type II collagen and proteoglycan (predominantly aggrecan) were demonstrated to be important for proper disc function, particularly in the nucleus pulposus (19). A previous study also demonstrated that significant changes in the extracellular matrix occur in the development of IDD (20). Consistent with the above research, our results suggest that downregulated miRNAs predominantly regulate pathways associated with the regulation of transcription by glucose and regulation of transcription by carbon catabolites.

In the current study, miRNA-222 levels were found to be upregulated in the IDD tissue and CDNK1B is one of the important target gene sets. A previous study demonstrated that miRNA-222 could directly target the 3'-UTR of CDKN1B and CDKN1C mRNAs to reduce CDN1B and CDN1C protein levels (21). The CDKN1B gene is a member of the Cip/Kip family of cyclin-dependent kinase (CDK) inhibitors that function to negatively control cell cycle progression (22), and coordinate competency for initiation of S phase with growth factor signaling pathways stimulating cell proliferation. In addition, deregulated miR-221 and miR-222 promote cancerous growth by modulating the expression of CDKN1B (23). The disturbed homeostasis induced by cell proliferation may contribute to the development and occurrence of IDD. Therefore, miRNA-222 may be a crucial element for IDD progression by inhibiting cell proliferation or pathways in cancer via targeting CDKN1B.

miRNA-589 levels are upregulated in the IDD tissue and the miRNA targeted SMAD4 in this study. It has been demonstrated that miRNA-589 mediates transforming growth factor (TGF) $-\beta$ induced epithelial mesenchymal transition in human 
peritoneal mesothelial cell, and epithelial mesenchymal transition is a key factor for the development and progression of peritoneal fibrosis (24). TGF- $\beta$, as an important proinflammatory factor, could promote the expression of catabolic genes and suppress the expression of critical extracellular matrix genes by the NP cells and AF cells in intervertebral disc tissue (25). Notably, SMAD4 is also a coactivator and mediator of signal transduction by TGF- $\beta$, which encodes a member of the Smad family of signal transduction proteins. Therefore, these results suggest that SMAD4 may be synergistic with TGF- $\beta$, and miRNA-589 may influence the extracellular matrix by targeting SMAD4. Although no experimental evidence has demonstrated that miRNA-589 targets SMAD4 in NP cells, the results of the present study provides further clarification.

In conclusion, the present study has provided novel information regarding the regulation network of IDD. The overexpression of miRNA-222 may be associated with IDD progression by inhibiting cell proliferation or pathways in cancer via targeting CDKN1B. Overexpression of miRNA-589 represses SMAD4 and influences the extracellular matrix via intracellular TGF- $\beta$ signal transduction. However, further studies are required to confirm the role of these miRNAs and target genes.

\section{References}

1. Kalichman L and Hunter DJ: The genetics of intervertebral disc degeneration. Familial predisposition and heritability estimation. Joint Bone Spine 75: 383-387, 2008.

2. Roberts S, Evans H, Trivedi J and Menage J: Histology and pathology of the human intervertebral disc. J Bone Joint Surg Am 2: 10-14, 2006.

3. Adams MA, Freeman BJ, Morrison HP, Nelson IW and Dolan P: Mechanical initiation of intervertebral disc degeneration. Spine (Phila Pa 1976) 25: 1625-1636, 2000.

4. Bartel DP: MicroRNAs: Genomics, biogenesis, mechanism and function. Cell 116: 281-297, 2004.

5. Khvorova A, Reynolds A and Jayasena SD: Functional siRNAs and miRNAs exhibit strand bias. Cell 115: 209-216, 2003.

6. Zhao B, Yu Q, Li H, Guo X and He X: Characterization of microRNA expression profiles in patients with intervertebral disc degeneration. Int J Mol Med 33: 43-50, 2014.

7. Wang HQ, Yu XD, Liu ZH, Cheng X, Samartzis D, Jia LT, Wu SX, Huang J, Chen J and Luo ZJ: Deregulated miR-155 promotes Fas-mediated apoptosis in human intervertebral disc degeneration by targeting FADD and caspase-3. J Pathol 225: 232-242, 2011.

8. Abouheif MM, Nakasa $T$, Shibuya $H$, Niimoto $T$, Kongcharoensombat W and Ochi M: Silencing microRNA-34a inhibits chondrocyte apoptosis in a rat osteoarthritis model in vitro. Rheumatology (Oxford) 49: 2054-2060, 2010.
9. Miyaki S, Nakasa T, Otsuki S, Grogan SP, Higashiyama R, Inoue A, Kato Y, Sato T, Lotz MK and Asahara H: MicroRNA-140 is expressed in differentiated human articular chondrocytes and modulates interleukin-1 responses. Arthritis Rheum 60: 2723-2730, 2009.

10. Smyth GK: Limma: Linear models for microarray data. In: Bioinformatics and computational biology solutions using $\mathrm{R}$ and Bioconductor. Springer pp397-420, 2005.

11. Benjamini Y and Hochberg Y: Controlling the false discovery rate: A practical and powerful approach to multiple testing. J R Stat Soc Series B Stat Methodol 289-300, 1995.

12. Nam S, Li M, Choi K, Balch C, Kim S and Nephew KP: MicroRNA and mRNA integrated analysis (MMIA): A web tool for examining biological functions of microRNA expression. Nucleic Acids Res 37: W356-W362, 2009.

13. Szklarczyk D, Franceschini A, Kuhn M, Simonovic M, Roth A, Minguez P, Doerks T, Stark M, Muller J, Bork P, et al: The STRING database in 2011: Functional interaction networks of proteins, globally integrated and scored. Nucleic Acids Res 39: D561-D568, 2011.

14. Smoot ME, Ono K, Ruscheinski J, Wang PL and Ideker T: Cytoscape 2.8: New features for data integration and network visualization. Bioinformatics 27: 431-432, 2011.

15. Maere S, Heymans K and Kuiper M: BiNGO: A Cytoscape plugin to assess overrepresentation of gene ontology categories in biological networks. Bioinformatics 21: 3448-3449, 2005.

16. Zhang B, Kirov S and Snoddy J: WebGestalt: An integrated system for exploring gene sets in various biological contexts. Nucleic Acids Res 33: W741-W748, 2005.

17. Duncan D, Prodduturi N and Zhang B: WebGestalt2: An updated and expanded version of the web-based gene set analysis toolkit. BMC Bioinformatics 11: P10, 2010.

18. Pattappa G, Li Z, Peroglio M, Wismer N, Alini M and Grad S: Diversity of intervertebral disc cells: Phenotype and function. J Anat 221: 480-496, 2012.

19. Le Maitre CL,Pockert A, Buttle DJ, Freemont AJ and Hoyland JA: Matrix synthesis and degradation in human intervertebral disc degeneration. Biochem Soc Trans 35: 652-655, 2007.

20. de Oliveira CP, Rodrigues LM, Fregni MV, Gotfryd A, Made AM and Pinhal MA: Extracellular matrix remodeling in experimental intervertebral disc degeneration. Acta Ortop Bras 21: 144-149, 2013.

21. Medina R, Zaidi SK, Liu CG, Stein JL, van Wijnen AJ, Croce CM and Stein GS: MicroRNAs 221 and 222 bypass quiescence and compromise cell survival. Cancer Res 68: 2773-2780, 2008.

22. Koff A: How to decrease p27Kip1 levels during tumor development. Cancer Cell 9: 75-76, 2006.

23. Wurz K, Garcia RL, Goff BA, Mitchell PS, Lee JH, Tewari M and Swisher EM: MiR-221 and MiR-222 alterations in sporadic ovarian carcinoma: Relationship to CDKN1B, CDKNIC and overall survival. Genes Chromosomes Cancer 49: 577-584, 2010.

24. Zhang K, Zhang H, Zhou X, Tang WB, Xiao L, Liu YH, Liu H, Peng YM, Sun L and Liu FY: MiRNA589 regulates epithelial-mesenchymal transition in human peritoneal mesothelial cells. J Biomed Biotechnol 2012: 673096, 2012.

25. Risbud MV and Shapiro IM: Role of cytokines in intervertebral disc degeneration: Pain and disc content. Nat Rev Rheumatol 10: 44-56, 2014. 